\title{
El golpe de Estado de 1973 y la intervención militar en la Universidad de Concepción (Chile)
}

\author{
Danny Monsálvez Araneda
}

Universidad de Concepción, Concepción, Chile

Email: dannymonsalvez@udec.cl

Mario Valdés Urrutia

Universidad de Concepción, Concepción, Chile

Email: mvaldes@udec.cl

\begin{abstract}
Resumen $^{1}$ : Se examinan los acontecimientos vividos en la Universidad de Concepción con motivo del golpe militar del 11 de septiembre de 1973 y sus consecuencias inmediatas: ocupación militar del campus universitario, suspensión de actividades académicas, reorganización universitaria, expulsión de funcionarios y estudiantes. Asimismo, son examinadas las medidas tendientes a finalizar el año académico tras el reinicio de actividades en noviembre.

Palabras clave: Universidad de Concepción, golpe de Estado, 11 de septiembre de 1973, dictadura.
\end{abstract}

\section{The 1973 military coup and the intervention at the University of Concepcion (Chile)}

Abstract: This article examines the events occurred in the Universidad de Concepción on the September 11, 1973 coup d'etat and its immediate consequences: military occupation of the University campus, suspension of academic activities, University reorganization, expulsion of staff and students. Measures to end the academic year after the restart activities in November are also examined.

Keywords: University of Concepción, coup d'etat, September 11, 1973, dictatorship.

\section{O golpe de Estado de 1973 e a intervenção militar na Universidade de Concepción (Chile)}

Resumo: Este artigo examina os acontecimentos vividos pela Universidade de Concepción com ocasião do 11 de setembro de 1973 e suas consequências imediatas: ocupação militar do campus universitário, suspensão de atividades académicas, reorganização universitária, expulsão de funcionários e estudantes. Assim mesmo, são examinadas as medidas tendentes a finalizar o ano académico trás o reinicio de atividades em novembro. 
Palavras-chave: Universidade de Concepción, golpe de Estado, 11 de setembro de 1973, ditadura.

\section{Introducción}

El presente artículose enmarca en la perspectiva historiográfica de la historia reciente como un campo en construcción. Es decir, con temas y problemas que aún están en desarrollo y donde se produce una relación directa entre el historiador y su objeto de estudio (Flanco y Levín, 2007; Bresciano, 2010 y Flier, 2014). Bajo esta óptica consideramos importante para el presente estudio explorar tanto los testimonios de quienes protagonizaron los hechos como las explícitas manifestaciones de control social evidenciadas en el periodo estudiado, especialmente con motivo de la coyuntura del quiebre social y político cuyo corolario fue el 11 de septiembre de 1973. Así, las entrevistas a testigos de los hechos, la documentación oficial del Estado y de la propia Universidad de Concepción, además de algunas informaciones deslizadas por la prensa nacional y local sometida a censura, constituyen el corpus central de las fuentes de información consultadas. Debemos además mencionar la falta de estudios e investigaciones sobre el tema en espacios micro-locales. Para el caso de Concepción, podemos mencionar nuestros trabajos y algunas tesis locales de pre y posgrado (Monsálvez, 2012, 2013, 2014a, 2014b y 2015; Valdés, 2006, 2012).

En el presente trabajo pretendemos analizar la intervención militar de la nueva autoridad política nacional nacida tras el golpe de Estado de 1973, en un espacio local, en este caso la Universidad de Concepción, la tercera universidad surgida en Chile en 1919. Esta institución educativa no era cualquier casa de estudios. Era la tercera universidad del país, había desarrollado un fuerte sentido de identidad y pertenencia regional. Por sus aulas habían transitado numerosos estudiantes y académicos que prestaron servicio a la región y el país. Asimismo, se había desarrollado desde los años sesenta una fuerte presencia del movimiento estudiantil, constituyéndose en un actor político de primer orden en la vida nacional. Por otra parte, existió un importante vínculo entre el Movimiento de Izquierda Revolucionaria (MIR) y el plantel penquista. De sus aulas salieron sus principales líderes y el primer rector elegido por votación triestamental, Edgardo Enríquez F., era el padre del líder del MIR, Miguel Enríquez. Conjuntamente, el Rector Enríquez se había desempeñado como Ministro de Educación del Gobierno de la Unidad Popular. Este tipo de hechos, fue configurando y proyectando la imagen de una "universidad roja”, que estaba a la vanguardia de los procesos sociales y políticos del país previo al golpe de Estado de 1973.

Enfocar aquí el análisis nos permite observar y responder las preguntas con respecto a qué ocurrió en la universidad penquista en el nuevo contexto nacional, junto con estudiar las particularidades producidas a raíz del proceso global de intervención en la educación superior chilena en los inicios de la dictadura cívico-militar. 
$\mathrm{Al}$ respecto, un primer eje de la nueva política universitaria emprendida en las casas de estudios superiores del país fue la llamada despolitización de la vida universitaria. Entendemos por este concepto el conjunto de medidas y/o acciones administrativas provenientes de la Junta Militar tendientes a apartar toda manifestación o expresión de naturaleza ideológica y política del trabajo académico llevado a cabo al interior de la universidad. Una vez excluidas las influencias político partidista que contaminaron según la Junta Militar- el quehacer y la convivencia universitaria, las instituciones de nivel superior debían volver a desarrollar las actividades que le eran propias: docencia, investigación y extensión universitaria. Lo anterior implicó la puesta en práctica de nuevas medidas de organización y gobierno universitario, apartando importantes grados de participación académica, funcionaria y estudiantil, tanto de la elección de las autoridades del plantel como de las prioridades académicas que debía atender la universidad. En el nuevo escenario que comenzó a modelarse desde el 11 de septiembre, cobró importancia la pronta política de intervención en las universidades a través del establecimiento de un Rector Delegado, nombrado desde el poder Ejecutivo establecido en Santiago, y sujeto solamente a su control; pues, duraría en su cargo por el tiempo que gozara de la confianza de la Junta Militar de Gobierno.

En vista de aquello, pretendemos comprobar que en el nuevo régimen gubernamental de facto surgido en 1973, la intervención militar tuvo como principal objetivo la reorganización institucional de las universidades, la respectiva depuración política en gran medida del quehacer de la universidad a través de los rectores delegados, para de esa forma prepararla para acercar sus actividades a formas de financiamiento con menor ayuda del Estado.

Dentro de las fuentes utilizadas (citadas al final del presente trabajo), se encuentran diversas entrevistas orales a testigos y protagonistas de los hechos examinados. Todos ellos, en su momento, consintieron en hacer públicas sus opiniones conservando sus nombres sin usar pseudónimos.

\section{La despolitización de la Universidad}

El día martes 11 de septiembre, las actividades en la Universidad comenzaron de manera tradicional, a las 8:00 de la mañana los estudiantes ingresaron a sus clases; sin embargo, con el transcurrir de los minutos, la comunidad universitaria se fue percatando del movimientos de tropas en la ciudad y de manera particular en el barrio universitario. La Universidad de Concepción fue rodeada desde temprano por efectivos del Ejército, los cuales instalaron ametralladoras en algunos cerros circundantes. Los edificios fueron ocupados en el transcurso de la mañana; lo propio aconteció con la mayor parte de los locales situados fuera del campus, caso de la Radio Universidad de Concepción. En las sedes de la Universidad ocurrió otro tanto (Valdés, 2006: 103-117). 
De esa forma comenzaba lo que diarioCrónica titularía como la “operación limpieza en la U”(Crónica, 15 septiembre de 1973: 2). ¿En qué consistió aquella "limpieza”? Tal como había estipulado el Bando número 3 en su punto 2, del 11 de septiembre de 1973, las clases y actividades académicas de la Universidad de Concepción, se suspendían por un periodo de 10 días. Escenario que se mantendrá hasta que "la situación nacional se halle totalmente normalizada especialmente en cuanto al orden público” (El Diario Color, 13 de abril de 1974). Si bien, se estipulaban 10 días, la reorganización de la Universidad, podría tomar más tiempo; no obstante aquello, las autoridades comenzaron a avanzar en las primeras medidas de corte administrativo.

Mientras Carabineros y Ejército ocupaban militarmente el campus universitario, se procedía al allanamiento de los hogares universitarios y al cierre del Departamento de Difusión, instancia a cargo del profesor Alejandro Witker. "Sus oficinas están cerradas”, mientras se procede a hacer una “limpieza” administrativa total(Crónica, 15 septiembre de 1973: 2). Asimismo -según consigna la prensa- los convenios que Witker había traído de Cuba y Moscú, fueron anulados. Horas más tarde Witker, junto a otros académicos y dirigentes políticos de la Unidad Popular local, eran trasladados al Estadio Regional e Isla Quiriquina.

Por otra parte, algunos directores de Escuelas fueron separados de sus cargos. Fue el caso del director subrogante de la Escuela de Periodismo, Enrique Echeverría. Su puesto ahora lo ocupó Carlos Godoy Rocca, quien semanas más tarde, pasará a ocupar el cargo de director de radio Universidad de Concepción. A la fecha, la Escuela de Periodismo era sindicada como uno de los principales focos del mirismo al interior de la Universidad (El Diario Color, 28 octubre de 1973: 7).

La radio universitaria fue ocupada y pasó a ser la Radio de las Fuerzas Armadas y Carabineros, identificándose así en sus transmisiones diarias. El control militar de la radio duró hasta septiembre de 1974. Hasta diciembre de 1973 hubo guardia del ejército en la planta transmisora, situado en el cerro La Gallina. El director de la radioemisora fue posteriormente separado de su cargo y se agregaron dos suboficiales como integrantes del equipo de la radio (Entrevista a Hugo Olea Morales y Carlos Godoy Rocca).

Otros de los cambios que se llevaron adelante, acontecieron en la Escuela de Servicio Social, su director, Luís Araneda, fue reemplazado en el cargo por la profesora Marta Montory (Cáceres, 2015: 40). Mismo caso ocurrió con aquellas unidades académicas que estaban dirigidas por personas “no adictas a la Junta Militar de Gobierno” (Crónica, 15 septiembre de 1973: 2). Otra de las escuelas que sufrió el rigor de la mano militar fue la de Sociología, básicamente por constituirse en un centro o espacio que sólo servía para la “subversión”.

Las personas retenidas en la isla lo fueron por razones políticas: tener militancia de partido vinculado a la Unidad Popular o a otros referentes de izquierda; pero respecto al personal universitario, al cual no había 
cargos de ninguna naturaleza que formular, fue paulatinamente liberado. Al contrario de lo ocurrido a otras personas vinculadas a la Universidad que no fueron liberadas y que permanecieron detenidas hasta un año en la isla Quiriquina, entre otros lugares de detención, sin formulación de cargos de ninguna especie (Entrevista a Sergio Ramón Fuentealba y a Carlos Godoy Rocca).

Para el caso de la Escuela de Periodismo, esta estaba conformada por dos departamentos: Periodismo y Comunicación. Alrededor de las 11:00 horas, fuerzas de Carabineros llegaron a esta escuela, procediendo a arrestar a todos los que se encontraban en su interior. En un bus de Carabineros fueron trasladados a la Base Naval de Talcahuano, desde donde fueron embarcados hacia instalaciones de la Armada en la isla Quiriquina (Valdés, 2006). Días más tarde, la Escuela fue allanada por efectivos del Servicio de Inteligencia Militar, de la Policía de Investigaciones y personal uniformado del Ejército. Según registro de prensa, en su interior se encontró "gran cantidad de propaganda subversiva, material de propaganda marxista y otras menudencias, como "cócteles molotov". Dicho operativo militar, se desarrolló la tarde del miércoles 3 de octubre de 1973. El material encontrado, estaba en una bodega, la cual permanecía cerrada, solo tenían acceso "algunos elementos docentes o estudiantes de la mencionada unidad. Con lo incautado se llenó un camión completo" (Crónica, 4 de octubre de 1973: 4). Como apunta Miguel Valderrama, para el campo de las Ciencias Sociales, "el proceso de represión política fue extremadamente duro. Se suprimieron unidades académicas, se clausuraron carreras, se congeló el ingreso de nuevos alumnos y se expulsó personal docente sobre la base de consideraciones exclusivamente políticas" (Valderrama, 2001: 5).

El día once, sólo una minoría pensó en resistir la acción militar. Un dirigente estudiantil de la época, Antonio Leal, Secretario de la Federación de Estudiantes, señalaba que había que resistir. Pero rápidamente fue arrestado y conducido a la isla Quiriquina; allí fue visto con el rostro golpeado (Entrevista a Sergio Ramón Fuentealba). En un reciente testimonio, Leal, recuerda que al momento del golpe de Estado era Secretario de la Federación de Estudiantes de la Universidad de Concepción. Al llegar al campus universitario, se percató que éste se encontraba ocupado por fuerzas militares. En medio de ese escenario, junto a otros dirigentes estudiantiles, intentó convocar a una asamblea en el foro de la Universidad, sin embargo, el control militar era total. La decisión fue entonces realizar un llamado a los estudiantes de izquierda para que abandonaran

"el barrio ya cercado militarmente e irse a las poblaciones populares para organizar una eventual resistencia (...) Esto ocurrió muy parcialmente porque los militares decretaron el estado de sitio y, muy temprano, el toque de queda, coparon las ciudades y controlaron los medios de comunicación”()

Si bien las informaciones son confusas respecto a las acciones de los partidarios de la Unidad Popular, “A esa altura nos dábamos cuenta que 
el Golpe, al menos en Concepción, era total y que el Ejército y la Marina controlaban las principales comunas” (Ibíd.).

Leal se refugió en la casa de un profesor de inglés del Instituto de Economía, quien vivía en el barrio universitario. Sin embargo, dicho sector fue registrado por los uniformados, procediendo a detenerlo. De ahí fue conducido a la Base Naval de Talcahuano y posteriormente a la Isla Quiriquina, lugares en los cuales fue torturado (Ibíd.).

Días más tarde, dos estudiantes ecuatorianos fueron encontrados acribillados en la ribera sur del Bío-Bío; perdieron la vida tras ser ejecutados después de haber estado acreditada su detención en la Cuarta Comisaría de Carabineros de Concepción. La prensa local afirmó tratarse de “extremistas”(Crónica, 25 de septiembre de 1973: 20). Cuarenta años después, la justicia dictó sentencia por este hecho, señalando que habían sido asesinados por personal de Carabineros (El Sur, 23 de octubre de 2013).

Entretanto el Rector Carlos von Plessing señaló que el año académico sólo estaba en receso, producto de la reestructuración interna que él estaba llevando adelante en la casa de Altos Estudios. Si bien hacia la semana del 20 de septiembre, ya se había constituido el Consejo Directivo de la Universidad, aún faltaba por designar las autoridades de cada Escuela o Instituto. Lo que si estaba en funcionamiento, era la parte administrativa. Además, se procedió a suspender 11 carreras en la Universidad: Economía Vespertina, Funcionarios del Poder Judicial, Ingeniería de Ejecución en Construcción Naval, Educación Básica en Matemáticas, Ciencias Naturales, Educación Media en Biología, Educación Media en Biología, vespertina, Educación Media en Matemáticas, Educación Media en Ciencias Naturales y, Asistente Dental Escolar; las cinco últimas correspondientes a la sede de Los Ángeles, además de Técnico Dibujante de Proyectos mecánicos de la sede del Carbón(El Sur, 23 de octubre de 1973: 9).

Un nuevo Consejo Directivo fue nombrado por rectoría para reemplazar en sus funciones al Consejo Superior de la Universidad. Quedó constituido por los siguientes académicos: Carlos von Plessing, Rector; Lorenzo González, Vicerrector; Humberto Otárola, Secretario General; René Ramos, Asesor Jurídico; Hervi Lagos, Tesorero; los profesores Hugo Tapia Arqueros, Enrique Oelcker, Gonzalo Peñailillo, Emilio Ginouvés, Luís Bardisa, Eleodoro Peña y Carmen Benavides; y, los estudiantes Ricardo Orellana, Roberto Torres, Jaime Campos y Hugo Larraín (Crónica, 25 de septiembre de 1973: 20).

A la fecha, las medidas dispuestas por el Rector von Plessing, contaron con el respaldo de la junta de comandantes a nivel local. En una declaración pública y en conjunto, el general Washington Carrasco Fernández y el contralmirante Jorge Paredes Wetzer, señalaron que ante la situación interna experimentada por la Universidad, las medidas de reorganización que está llevando adelante el Rector y en base al "principio de autonomía universitaria”, corresponde que su autoridad "estudie y resuelva las medi- 
das que sean necesarias para reorganizar íntegramente la señalada Corporación”; por lo tanto, las autoridades comparten y respaldan las medidas dispuestas por la máxima autoridad universitaria (El Sur, 22 de septiembre de 1973: 9).

Pero no todo parecía represión o violencia por aquellos días al interior de la Universidad. La nota "llamativa” dada a conocer por un medio de prensa local, daba cuenta de un académico universitario que se había quedado sin sueldo. El abogado Mario Muñoz, había perdido una apuesta con su colega, Humberto Otárola, lo cual le habría significado quedarse sin sueldo durante el mes de septiembre. ¿En qué consintió la apuesta? Supuestamente, durante agosto, Otárola le comentó a Muñoz “que los militares asumían el Gobierno antes del 30 de septiembre. Muñoz afirmó que sería después de esa fecha”. El resultado es por todos conocidos. Si bien Muñoz perdió la apuesta estaba "feliz con el resultado". "Sabe que si los uniformados hubieran esperado hasta después del 30 de septiembre, ninguno de los dos habría ganado la apuesta. La habrían perdido ambos, y uno de ellos, Otárola, la cabeza. Estaba en la lista”, en clara alusión al "Plan Z” (Crónica, 29 de septiembre de 1973: 16); un supuesto plan de eliminación de los principales opositores al gobierno de la UP, el cual resultó ser falso (Monsálvez, 2015).

Con todo, la Universidad de Concepción no sufrió los rigores de la violencia que experimentó en Santiago la Universidad Técnica del Estado (Gómez, 1995: 89). Para el caso de esta última, con su sede en Concepción, la meta de las nuevas autoridades era "limpiarla" y darle la imagen de Universidad. Esas fueron las palabras emitidas por el nuevo vicerrector académico, sede Concepción, Víctor Normandi Vega y la secretaria Cristina Toro de la Fuente, los cuales habían sido ratificados en sus cargos por parte del Rector - Delegado de la Universidad estatal, Coronel Eugenio Reyes (Crónica, 7 de diciembre de 1973: 20).

Un segundo momento de detenciones por razones políticas ocurrió en la Universidad el día de cancelación de sueldos a sus funcionarios. El pago tuvo lugar entre las 10 y las 13 horas del jueves 4 de octubre en la Biblioteca Central. Al momento de salir los funcionarios, tras cobrar su cheque, eran retenidos por algunos sujetos que comprobaban su identidad; quienes figuraban en una lista que portaban aquellos, eran detenidos en ese momento y posteriormente enviados a centros de detención.

Así, entre el 11 de septiembre y el 4 de octubre de 1973, un número significativo de estudiantes, académicos, funcionarios y empleados universitarios fueron trasladados en camiones militares hacia diversos lugares de detención (Entrevista a Pedro Ilabaca y Dagoberto Ulloa). Uno de los detenidos por aquellos días fue el ex vicerrector Galo Gómez, quien fue conducido arrestado en un vehículo de la universidad (Verdugo, Riquelme, Soto y Varela, 2000). Trasladado a la Isla Quiriquina, posteriormente fue traído al continente, específicamente a su domicilio particular. Sin embargo, efectivos de Investigaciones lo trasladaron al cuartel de la policía civil para 
ser interrogado. Galo Gómez, había sido en su época de estudiante, presidente de la Federación de Estudiantes de la Universidad de Concepción. Luego regidor de Concepción y al momento del golpe de Estado se desempeñaba como presidente de la Comisión Nacional de Investigación Científica y Tecnológica (Conicyt). Además, era un destacado militante del Partido Socialista (PS) en la zona.

Otros de los detenidos por aquellos días fue el Dr. Jorge Peña Delgado, académico de la Escuela de Medicina de la Universidad, ex director zonal de la novena Zona del Servicio Nacional de Salud y amigo personal de Salvador Allende. A Peña, se le sindicaba y vinculaba -entre otras cosascon el ocultamiento de armas que realizó el PS, así como, haberse "escapado” al extranjero con dineros del Servicio Nacional de Salud (El Sur, 27 de septiembre de 1973: 3). Efectivamente, al momento del golpe, Jorge Peña se encontraba en el extranjero, realizando algunas gestiones para adquirir material para la repartición donde se desempeñaba como jefe. Días después, decide regresar a Chile, momento en el cual es detenido por personal uniformado. A Peña Delgado, se sumó Pablo Dobud, militante socialista y ex profesor de la Escuela de Ingeniería de la Universidad de Concepción(Crónica, 2 de octubre de 1973: 5).

Pero no eran los únicos docentes detenidos. A todos ellos, se les asoció o vinculó con acciones del depuesto gobierno de la Unidad Popular. "Entre los afectados por la acción se cuenta el abogado y ex - director de la Escuela de Leyes de la Universidad de Concepción, Sergio Jarpa Fernández y varios miembros del Consejo Superior de esa Casa de estudios” (Crónica, 4 de octubre de 1973: 4).

Las listas de personal para detener fueron confeccionadas por la inteligencia militar. En ello hubo colaboración civil proveniente de diversos sectores, incluyendo personal de la propia universidad, que actuó como “informante” a título personal (Entrevista a Carlos Godoy Rocca).

Un fenómeno que aconteció a raíz de la nueva situación política creada fue la diversidad de denuncias anónimas provenientes de personal universitario en contra de otros funcionarios. Unos denunciaban al docente "N" quien supuestamente ocultaba armas en su casa. Otros denunciaban al funcionario " $M$ ” supuestamente involucrado en acciones extremistas. Hasta hubo denuncias risibles: la persona del rector fue denunciada anónimamente de distribuir alcohol de quemar y bencina blanca para elaborar bombas molotov con las cuales atacar las patrullas militares.

Lo cierto es que la Universidad fue vista, por parte de la autoridad militar, como un "foco ultra”, desde fines de la década del sesenta hasta el 11 de septiembre de 1973; donde el Movimiento de Izquierda Revolucionaria (MIR), había convertido a la Universidad "en el principal foco de concientización marxista y de penetración política, especialmente en los años en que le correspondió ocupar el cargo de rector a 
Edgardo Enríquez Frodden y la Vicerrectoría a Galo Gómez Oyarzún”. Este último sería "la eminencia gris tras el trono” (Crónica, 3 de octubre de 1973: 4). Pero no era todo, la radio de la Universidad, también había caído bajo control de los marxistas. Su primer director fue el comunista Mario Céspedes, con lo cual dicho espacio habría sido copado por militantes de ese partido.

Relacionado con el tema periodístico, la Escuela de Periodismo de la Universidad, prontamente fue controlada por elementos del MIR, quienes comprendieron la importancia que tenía el periodismo para una labor de concientización de masas. A ojos de los partidarios del golpe de Estado, esta unidad académica, pasó a constituirse en un feudo mirista y socialista. Mismo caso corría para Sociología y Economía.

La presencia y acción de extranjeros, fue otro de los elementos que contribuyeron a reforzar el proceso de concientización marxista al interior de la Universidad. Muchos de ellos

“Entraban por la ventana. Sin concurso. La gran mayoría, argentinos, uruguayos, brasileños, mexicanos y colombianos. No eran los únicos, "también se concedieron becas a extranjeros, en los últimos años a muchos cubanos. Aparte de los estudios se les pagaba su estadía en Concepción, donde vivían a cuerpo de rey, en los mejores hoteles céntricos” (Ibíd.).

Sobre la presencia de extranjeros en la Universidad, el general Washington Carrasco ha señalado que después del 11 de septiembre, él personalmente trasladó en camiones militares a un grupo de "profesores extremistas de la Universidad de Concepción”, unos 15 o más tupamaros y montoneros al paso internacionalPajaritos, hoy Cardenal Samoré, desde donde se les expulsó del país (La Segunda, 5 de septiembre de 2003: 11).

Todo este cuadro “ultra”, se completaba con una Federación de Estudiantes controlada por miristas, socialista y comunistas. Cuyo actuar estuvo caracterizado por abusos y escándalos, relacionados con dineros, de los cuales nunca dieron cuenta.

Con todas estas acciones, la Universidad se había convertido en un “foco de subversión”, en el cual muchos docentes e investigadores se prestaron para este juego. En palabras del recién designado rector-delegado Guillermo González Bastías:

“...ellos se olvidaron del verdadero rol que debe tener una Universidad. Nosotros, ahora lucharemos por elevar la condición del universitario y para ello vamos a mantener un verdadero pluralismo ideológico. Vamos a limpiarla de elementos que la usaron exclusivamente para desprestigiarla” (Crónica, 3 de octubre de 1973, p. 4). 


\section{Entre la intervención y la reorganización}

El martes 11 de septiembre, un auxiliar de la Casa Central avisó al rector del movimiento militar en el país. Después de comunicarse con diversos colaboradores, el rector concurrió al cuartel general de la III División de Ejército, donde se reunió con el General Washington Carrasco, en esos momentos, la máxima autoridad defacto existente en Concepción. La entrevista tuvo lugar bien avanzada la mañana. Encontrándose el general bastante inquieto, pues, aún se desarrollaban las acciones militares en el centro de Santiago. En concreto, el rector le pidió al general Carrasco prudencia, pues, no quería ningún hecho luctuoso en la universidad. Hecha la petición, se marchó (Entrevista a René Ramos Pazos).

Con las clases suspendidas, gran parte de los edificios cerrados, la vigencia de un toque de queda y frente al cese de hecho de los cuerpos colegiados de la corporación, von Plessing decidió declarar en reorganización total a la Universidad y formó un Consejo Asesor para que colaborasen con su gestión. En este aspecto, hubo peticiones claras por parte del Almirante Jorge Paredes Wetzer, Comandante de la II Zona Naval. Pidió a von Plessing tomar medidas en la Universidad en relación con Periodismo y con el Teatro Universitario. La autoridad militar provincial pidió al Rector un listado de los extranjeros que trabajaban en la universidad (El Diario Color, 25 de septiembre de 1973: 1 y 9).

En consecuencia, en el nuevo escenario surgido tras el once de septiembre, el rector dio a conocer el jueves 20 los alcances de la reorganización, la cual era mucho más amplia que los aspectos puntuales solicitados por la Junta Militar.

A lo ya dicho por von Plessing, en el sentido que el año académico estaba en receso, consideró cinco puntos como sustento de su determinación. Primero, invocó que al realizarse la elección de rector y vicerrector en 1972 (donde resultó ganador), planteó que introduciría "profundas modificaciones" destinadas a hacer de la Universidad de Concepción una institución de educación superior que cumpliera sus estatutos. Segundo, que la comunidad universitaria "al elegirnos por amplia mayoría en democrática elección” hizo suyos esos propósitos. Tercero, que el Consejo Superior no puede considerarse representativo de la comunidad universitaria por el sistema de su elección; y, que, al no estar aprobada una Política General, pese a haber transcurrido cinco años desde la aprobación estatutaria de la Universidad, "no ha existido la fuente de donde emana la competencia del Consejo Superior"; además del hecho de haber entrabado la acción de las nuevas autoridades [elegidas en 1972]. Cuarto, que por lo anterior, la autoridad universitaria dispuso elaborar un documento de "rectificación universitaria que aspiraba a someter a la decisión de un Claustro Pleno" que convocaría en octubre. Finalmente, en quinto lugar, consideraba que dadas "las actuales circunstancias extraordinarias por las que atraviesa el país no permiten la realización del Claustro Pleno”; en consecuencia, resolvió: 
"1.- Asumir el poder pleno universitario con el fin de tomar con prontitud todas las medidas indispensables para la conservación del orden y disciplina y el prestigio de la Universidad, como única manera de normalizar las actividades universitarias e integrar a esta Casa de Estudios en una política de realidad nacional;

2.- (...) para (...) alcanzar los objetivos que señala el artículo $1^{\circ}$ de los Estatutos, se disuelven el Consejo Superior y los demás organismos colegiados de la Universidad de Concepción y cesan en sus cargos sus autoridades.

3.- El rector ejercerá el poder pleno universitario asesorado por un organismo que se denominará Consejo Directivo, que integrarán el Vicerrector Lorenzo González Coppola; el secretario general, don Humberto Otárola Aqueveque y las demás personas que el rector designe.

4.- En mérito al poder que he asumido declaro en reorganización total la Universidad. Todos, el personal docente, administrativo y de servicio pasa a tener la calidad de interino 5.- Suprímese el Instituto de Sociología y la Escuela de Periodismo por resultar evidente y de público conocimiento que permanentemente han ofendido el espíritu y objetivos universitarios. Declárase en consecuencia, la caducidad de los contratos de trabajo de todo el personal docente que prestaba servicios en estas unidades. El personal administrativo y de servicios quedará en las condiciones señaladas en el punto anterior.

6.- Todos los estudiantes de la Universidad, para conservar esta calidad deberán matricularse de acuerdo con las normas y en el período y lugares que se indicarán oportunamente.

7.- (...) los hogares universitarios permanecerán cerrados con el objeto de permitir su total reorganización.

8.- Todos los elementos de difusión con que cuenta la Universidad (radioemisora, cine, imprenta, etc.) quedan a disposición de esta Rectoría y su personal deberá ajustarse estrictamente a las normas e instrucciones que la autoridad universitaria imparta. Cualquier contravención (...) traerá como consecuencia la caducidad del contrato de trabajo.

9.- Todos los extranjeros que a la fecha laboraban en la Universidad (...) incluyendo (...) alumnos, deberán, dentro del plazo de 15 días (...) registrarse personalmente en la Secretaría General de la Universidad, premunidos de todos los antecedentes de extranjería debidamente visados por las autoridades competentes, con sus contratos de trabajo y una declaración por escrito, firmada ante Notario, en la que se precise títulos y antecedentes académicos del país de origen 
y funciones que desempeñaban a la fecha en la Universidad de Concepción.

10.- Todo lo indicado anteriormente afecta asimismo a las sedes de la Universidad.

11.- Oportunamente, el rector propondrá un nuevo proyecto de estatutos por los cuales se regirá la Universidad de Concepción” (En Archivo General de la Universidad de Concepción. Inserción universitaria en El Sur, 21 de septiembre de 1973: 1).

Cuatro días después del decreto de reorganización, se constituyó el Comité Directivo (ya indicado) que reemplazó al Consejo Superior. Una vez instalado, el comité comenzó a desarrollar dos tareas prioritarias, examinar la situación de docentes, no docentes y alumnos extranjeros y preparar la designación de directores y secretarios de cada una de las unidades académicas de la universidad (El Diario Color, 25 de septiembre de 1973: 9). Asimismo, analizar el tema de las apelaciones de aquellos alumnos que consideran que cumplían con los requisitos, pero que por errores han sido marginados de las listas (Crónica, 29 de octubre de 1973: 20).

El Rector von Plessing señaló entonces que se respetaría el pluralismo ideológico en la Universidad de Concepción. No se sancionaría a nadie por el solo hecho de tener una posición política determinada, marginándose de la Casa de estudios a quienes estuvieran comprometidos en hechos punibles por la justicia. En efecto, afirmó: "El pluralismo se respetará; no habrá revanchismo ni venganzas personales, pero todos los docentes y no docentes que aparezcan comprometidos en hechos estimados punibles por la justicia, serán marginados de la Universidad”(El Diario Color, 25 de septiembre de 1973, portada). Luego agregó:

“A la Universidad se viene a estudiar y a enseñar; pero no a hacer agitación. Aquellos que no estén comprometidos en actos subversivos o terroristas o sencillamente ilegales, en cuanto al cumplimiento de las funciones para las cuales fueron contratados por la Universidad, nada tienen que temer” (Ibíd.)

\section{Y añadió:}

"Se quiere causar el menor trastorno posible con la reorganización, pero esto no debe entenderse como flaqueza o arrepentimiento por parte de la autoridad, sino porque nos interesa devolver la normalidad académica y general a la Universidad cuanto antes. El que quiere hacer la revolución, tiene que asumir después las responsabilidades que de ello se desprendan” (Ibíd.).

Por otra parte, von Plessing no tenía intenciones de "seguir por mucho tiempo con plenos poderes". Le interesaba "restaurar pronto la normalidad, con los organismos de conducción que se acuerde en el [futuro] 
Claustro". En cuanto a las clases, pensaba que "el 5 de noviembre podría retomarse el desarrollo del segundo semestre de 1973 que finalizaría en febrero de 1974" (El Diario Color, 25 de septiembre de 1973: 9). El regreso a la normalidad implicaba que proseguirían actuando los organismos estudiantiles, con una reglamentación previamente elaborada "para evitar malos entendidos". No se desconocía que los alumnos podían tener sus propias y diversas posiciones "pero no podrán usar de ellas para fines extrauniversitarios” (El Diario Color, 26 de septiembre de 1973: 4).

Entretanto el 25 de septiembre, el Rector de la Universidad de Chile, Edgardo Boeninger, junto a dos vicerrectores visitó la Junta Militar de Gobierno, ocasión en la cual expuso entre otros aspectos, la reestructuración de las universidades, la competencia que tendrá en esta la autoridad militar y normas de orden general. Al respecto,

“se le pide al Sr. Rector que haga llegar un memorándum escrito de la pauta tratada a fin de que sea estudiada a fondo por la Junta y llegar a un determinación clara sobre el problema, expresándoles además que será de interés especial del Gobierno las Universidades, pero que básicamente es preciso contar con orden y disciplina interna” (Acta número 9, 25 de septiembre de 1973).

Dos días más tarde, la Junta Militar acordó citar a la mayor brevedad al Consejo de Rectores (Acta número 10, 27 de septiembre de 1973). En dicha reunión de la Junta, Augusto Pinochet manifestó que

“en definitiva el gobierno decretará en reorganización las Universidades, se nombrarán Rectores - Delegados del Gobierno con plenos poderes en cada plantel universitario los que asumirán sus funciones dentro de los 10 próximos días quedando los actuales Rectores en carácter de dimisionarios y se estudiará una nueva legislación al respecto para aplicarla en el más breve plazo” (Ibíd.)

El resto de los integrantes de la Junta, respaldó los comentarios y determinación de Augusto Pinochet, agregando que "se espera que este período de transición durará lo que se precise hasta tener totalmente saneada la administración de las diversas Universidades y que en ningún caso ello significará persecuciones ideológicas, salvo para aquellos elementos y/o teorías marxistas y extremistas” (Acta número 11, 28 de septiembre de 1973).

¿Cuál fue la reacción de los rectores? Según el acta de la Junta Militar, los rectores acataron la determinación, ofreciendo su cooperación en sus respectivas especialidades, pero veían con preocupación, temor e inquietud la tarea que llevarán adelante las autoridades militares, ya que era una labor muy "ardua y difícil” y “que era su interés personal, realizar ellos la dura tarea de reorganización y limpieza dentro de sus planteles” (Ibíd.)

Boeninger resumió escuetamente el parecer de los rectores universitarios: "Lo acato, pero lo lamento" (El Diario Color, 30 de octubre de 1973:12). 
Fue el único comentario que deslizó tras la reunión. No agradó a los rectores la decisión gubernamental. Posteriormente, en Concepción, von Plessing no ocultó su pensamiento ni emoción frente a estos acontecimientos. En una ocasión expresó muy emocionado: “pobre Universidad; quizás en qué manos va a caer” (Entrevista de Hervi Lagos Cortés). La preocupación y congoja de von Plessing acaso ocurría porque ¿esperaba que la Junta Militar lo ratificara en el cargo, dentro del nuevo orden? O su preocupación era porque la Universidad podría pasar a ser conducida por personas ajenas a la academia. Nuestra hipótesis es que von Plessing estaba esperanzado en que la Junta lo ratificara en el cargo, considerando las medidas que había tomado inmediatamente producido el Golpe, y atendiendo al hecho de que no era de izquierda y había sido electo en 1972 por una mayoría universitaria opositora al gobierno del presidente Allende.

Para von Plessing la resolución gubernamental de intervenir las universidades significó el cese en el ejercicio de su cargo. El rector declaró que había presentado su renuncia a la Junta Militar en la reunión del 29 de septiembre(El Sur, 2 de octubre de 1973: 9). Más o menos formalidades, la verdad sin eufemismos: don Carlos fue abruptamente cesado por el gobierno militar en la rectoría de la Universidad de Concepción, cargo para el cual había sido elegido por un periodo de cuatro años. Los demás rectores sufrieron la misma medida, no hubo excepciones.

\section{La “mano militar”: el primer Rector-Delegado}

El 1 de octubre de 1973, el Capitán de Navío (R) Guillermo González Bastidas, asumió como Rector - Delegado la conducción de la Universidad de Concepción. El nuevo rector era una persona que había trabajado como funcionario administrativo en la universidad, y había sido presidente de la Asociación del Personal universitario, reelegido con la primera mayoría en el mes de agosto. Un hombre que concitaba mucha adhesión en la universidad, pero con un pecado original: “no pertenecía al ámbito académico” (El Sur, 14 de octubre de 1973: 1).

Frente a una pregunta periodística con respecto al modelo de universidad que impulsaría la nueva autoridad, y de qué manera se opondría a los conceptos de militante y comprometida, González respondió:

“El pluralismo ideológico se opondrá al concepto de comprometida y militante. Yo creo en una universidad comprometida con el conocimiento. Su deber es entregar, difundir y crear conocimiento con respecto a todas las ideologías que respeten el pluralismo filosófico. La libertad de conocimiento, atributo y derecho del hombre desde que nace, por lo tanto entregado a Dios, debe respetarse” (Ibíd.).

Una de las primeras medidas del nuevo Rector, fue designar dos nuevos vicerrectores, se trataba del profesor y ex director de la Escuela de Ingeniería, Mario Olavarría, quien a la fecha se desempeñaba como delega- 
do del Rector en las áreas de Ciencias Físicas, Matemáticas y Química. Olavarría también había sido regidor de Concepción, elegido en abril de 1971, en representación del Partido Nacional. El otro vicerrector, administrativo, era Hervi Lagos, quien se desempeñaba como tesorero general de la Universidad. A estas designaciones, se sumaron las de Gustavo Pizarro Castro, como delegado para la sede del Carbón y Juan de Luigi Lemus, como delegado - director de la Biblioteca Central (El Sur, 19 de octubre de 1973: 4).

González Bastías procedió a crear un Consejo Directivo con base en una división por áreas de la Universidad. Cada una de estas áreas tuvo un delegado. En total fueron cinco las áreas que se estipularon:Salud, Ciencias Sociales, Física y Matemáticas, Asuntos Estudiantiles, Matrícula y Registro y, Área Administrativa. La presencia estudiantil quedó descartada.

A fines del mes de octubre, el Rector-Delegado se reunió con los periodistas locales, en la ocasión informó que la Universidad normalizaría “todas sus actividades” (El Diario Color, 25 de octubre de 1973: 7). Para el caso de los alumnos de las carreras de sociología y periodismo, las cuales fueron cerradas, éstos tendrán la posibilidad de continuar sus estudios en otra universidad o en otras carreras de la propia universidad. En otros ámbitos del quehacer universitarios, se refirió a la clausura del Teatro de la Universidad, señalando que se había adoptado dicha medida "porque no estaba desempeñando las funciones para lo que fue creado. Pero ello no significa que esté definitivamente cerrado. Habrá posibilidades más adelante, que estudiamos” (El Diario Color, 25 de octubre de 1973: 7). El personal del Teatro, fue apartado de sus funciones en la Universidad.

Entretanto, la Universidad buscó reubicar a los estudiantes y profesionales que habían quedado sin sus lugares de estudio y trabajo a consecuencia del cierre de sus carreras, escuelas, departamentos o institutos. En el caso de los docentes que tuvieron que dejar sus cargos, esto se debía señaló - a la "nefasta influencia que ejercían sobre el alumnado, siendo responsables en gran medida de haber incitado a los jóvenes a actividades contrarias a la sociedad, recurriendo a la violencia y quebrando la convivencia universitaria y de la comunidad” (Ibíd.). Sin embargo y a pesar de sus acciones y actitudes, "se les han respetado íntegramente sus beneficios, en especial los previsionales, de tal manera que no han sufrido daño económico” (Crónica, 30 de octubre de 1973, portada).

Los estudiantes que no figuraron en las listas de quienes se les permitía rematricularse, fueron apartados por razones políticas y por falta de créditos. Para volver a matricularse, los alumnos de primer año debía tener aprobados 6 créditos en el primer semestre más el curso de nivelación; los de cursos superiores, debían tener aprobados 9 créditos en el primer semestre. Hubo también un proceso de apelación al cual pudieron acceder los afectados (El Sur, 23 de octubre de 1973: 9). En síntesis, de los 18.000 alumnos de esta universidad pudieron volver a matricularse 10.000 estudiantes, es decir, el 56\% del total del alumnado; el 44 \%o volvió a la 
Universidad de Concepción (El Sur, 29 de octubre de 1973: 9). El 29 de octubre comenzarían los alumnos a rematricularse y el 5 de noviembre retornaron a clases. Mientras que en materia docente, el criterio seguido por la autoridad en esta materia fue principalmente político. Todo académico y funcionario que tuvo militancia visible en algún partido de la UP o en el Movimiento de Izquierda Revolucionario, fue separado de la corporación. En la época en estudio, parte de los académicos - y la autoridad - que compartían una visión de las cosas donde era necesario serenar los espíritus y separar de la universidad las voluntades inspiradas en el marxismo, partidarias de la revolución, consideraron el alejamiento de profesores y funcionarios como algo necesario. Para los afectados, la exoneración de personal era una arbitrariedad (Entrevistas a Pedro Ilabaca G. y a Fernando Antinao J.). En cuanto a las carreras universitarias, recordemos que once de ellas fueron suspendidas. A fines de octubre, un aviso por la prensa local llamaba a presentarse a trabajar a todos aquellos que aún no lo hacían.

Por aquellos mismos días, se daba a conocer la estructura definitiva que tendría la Universidad de Concepción, en cuanto a nombres y cargos de las nuevas autoridades. La encabezada el Rector-Delegado Guillermo González Bastías, Mario Olavarría como Vicerrector Académico y Hervi Lagos como Vicerrector Administrativo, mientras que Ramón Domínguez Águila, ocupaba el cargo de Secretario General. Luego estaba un Consejo Consultivo Asesor, integrado por seis académicos, cada uno de ellos como delegado de área. Finalmente estaban los Directores de Unidades y Jefes de Reparticiones.

El lunes 5 de noviembre, todos los alumnos debían presentarse a clases. Así lo informó el Rector-Delegado a través de un comunicado por la prensa, señalando que el segundo semestre académico, comenzaría aquel día. Igualmente, se comunicó que el periodo de re matrícula para cursar el segundo semestre, se daba por terminado, mientras que las apelaciones "serán resueltas en única instancia por el rector-delegado, Guillermo González Bastías, una vez que sean debidamente informadas y verificadas por el área Estudiantil y por la respectiva unidad en que el alumno cursaba sus estudios” (El Diario Color, 3 de noviembre de 1973: 7).

Horas más tarde, diario El Sur informó que tras el primer día de clases un "grato ambiente reina en la U". Al ser elegidos al azar un grupo de estudiantes, todos expresaron su satisfacción por el retorno a clases, y al mismo tiempo apreciaron la tranquilidad y el orden que ahora reinaba en la Universidad. Se veía limpia, se puso término a la propaganda política y afiches que llamaban al odio. Es decir, "Los universitarios entrevistados por El Sur se sentían felices de estar nuevamente con sus compañeros y en todos los rincones del Barrio Universitario se veían grupos que conversaban y reían luego de volver a encontrarse” (El Sur, 6 de noviembre de 1973: 9).

El jueves 8 de octubre a las 19:30 horas en el Teatro Concepción y ante la presencia de las más altas autoridades locales, es decir, los representantes de la "Honorable Junta Militar de Gobierno", Jefe de la Cuarta Zona 
de Inspección de Concepción del Cuerpo de Carabineros, Silvio Salgado; el presidente de la Corte de Apelaciones de Concepción, Carlos Cerda; el alcalde de Concepción, Alfonso Urrejola; el prefecto Jefe de Concepción, Coronel Benjamín Bustos; el vicerrector delegado de la Universidad Técnica del Estado, mayor de Carabineros, Carlos Gallegos, representantes de medios, directores de escuelas e institutos de la Universidad, personal administrativo y alumnos, el Rector-Delegado de la Universidad penquista, Guillermo González Bastías, reanudó las actividades académicas de la Universidad. Sus primeras palabras apuntaron a señalar que la Universidad iniciaba una nueva etapa de su vida, "es como si dijéramos que estuvo mucho tiempo enferma y ahora, en plena convalecencia, exige revitalizarse para seguir cumpliendo en plenitud sus nobles objetivos” (ibíd.). Que en su momentos, la Universidad, era una de las más respetada del país, pero que en los últimos años "llegó a convertirse en la más conflictiva de Chile, más aun, de Latinoamérica, llegando a recibir el epíteto de "La Universidad Roja” de este continente" (Ibíd.), sin embargo, gracias a las Fuerzas Armadas, el país y las Universidades emprenden un nuevo rumbo, dejando atrás, momentos complejos y oscuros para Chile y su gente. Que en el caso de los institutos armados, "conscientes de su alto deber y de su alta misión y atendiendo el clamor de no menos del 80 por ciento de la población de nuestro país, se han evidenciado como la gran reserva moral de nuestro suelo" (Ibíd.).

Terminó su discurso, dirigiendo un mensaje a los profesores y sobre todo a los alumnos a quienes hizo un llamado a que "se dediquen a los estudios, porque el saber será lo único que le dará confianza en el andar de un camino que debe tener como única meta la alegría de vivir” (El Diario Color, respectivamente, 9 de noviembre de 1973, portada; Diario el Sur, 9 de noviembre 1973: 9)

\section{Comentarios finales}

Dentro de las investigaciones que se han realizado sobre el golpe de Estado de 1973 y los inicios de la dictaduracívico-militar, destacan de preferencia aquellos que abordan la represión militar contra la población. Una importante cantidad de ellos centrados en Santiago, con lo cual los espacios locales o regionales quedan invisibilizados, omitidos o bien se tiende a homologar lo ocurrido en la capital con el resto del país. Sin embargo, resulta importante cambiar el foco de análisis para adentrarse en lo ocurrido tras el golpe militar en espacios micro-locales. Precisamente ese fue el objetivo central de lo señalado en las páginas del presente artículo, dar cuenta de la intervención militar ocurrida a nivel universitario, especialmente en regiones. Es el caso de lo acaecido en la Universidad de Concepción.

Aquel martes 11 de septiembre de 1973, la Universidad penquista, al igual que gran parte del país, sufrió la ocupación militar de sus espacios e instalaciones. Se procedió a la suspensión de las clases, el allanamientos de los hogares estudiantiles y el posterior arresto por parte del personal militar 
y del cuerpo de Carabineros de un número indeterminado de académicos, funcionarios administrativos y estudiantes, los cuales con el transcurrir de las horas y días, fueron trasladados a los diversos recintos de detención, con el objetivo de ser interrogados, proceso en el cual varios de ellos fueron víctimas de torturas.

De esta forma, se dio inicio a uno de los periodos más complejos, difíciles y oscuros de la vida en la Universidad de Concepción, en el cual "el desarrollo libre del espíritu” quedó suspendido o supeditado a la vigilancia y el control del gobierno militar de facto sin contrapeso alguno.

El golpe de Estado significó para la Universidad de Concepción el término de la Universidad reformada, esfuerzo institucional iniciado en 1968. Pero también significó -con la prohibición del derecho a reunión- que el rector von Plessing se viera obligado por la fuerza de los hechos a asumir el control y la reorganización de la universidad, dentro del nuevo escenario nacional conducido por la Junta Militar de Gobierno surgida con apoyo civil, tras el 11 de septiembre de 1973. Los cambios institucionales que se impulsaron desde la primera hora, más la profundización del esfuerzo por depurar la universidad de influencias y actores políticos y revolucionarios, corrió además por cuenta del Rector-Delegado: cierres adicionales de carreras, exoneraciones, alejamiento de estudiantes, implicaron cambios y remezones en la vida académica que no se veían desde la época de la reforma universitaria indicada, interrumpida en 1973.

La imagen de "Universidad roja” que se tenía de la casa de estudios, por la presencia de importantes sectores y grupos de izquierda, entre ellos el MIR, significó que una vez producido el golpe militar, las Fuerzas Armadas intervienen con prontitud y rapidez en la Universidad. ¿El objetivo de aquella intervención? Ejercer un rápido control sobre el mundo de la academia, especialmente sobre aquellas que a vista de la Junta Militar se habían constituido en espacios que albergaron durante el gobierno de Salvador Allende a grupos y sectores políticos e ideológicos vinculados al marxismo. En ese grupo estaba la Universidad de Concepción. En ese contexto se enmarca y entenderá el proceso de despolitización y desideologización llevado adelante por los Rectores-Delegados militares.

En consecuencia, adentrase en el estudio de lo que fue la intervención militar en las universidades tras el 11 de septiembre de 1973, el proceso de reestructuración institucional en el mundo de la academia, la designación de rectores delegados y la depuración que estos impulsaron en los diversos planteles universitarios, constituyen un interesante campo de estudio para analizar el poder disciplinario y normativo que tuvo el golpe de Estado de 1973, pero a nivel de espacios locales, específicamente en el mundo de las universidades; particularmente de aquellas que eran de provincias, como el caso de la Universidad de Concepción.

Debemos remarcar que las medidas impuestas por la junta militarbuscaron despolitizar el ambiente académico, léase, prohibir y apartar 
toda actividad política-partidista de las universidades; y por otra parte, impulsar un proceso de desideologización entendido este como eliminar de la universidad a los seguidores de la ideología marxista en sus distintas expresiones, las que habían permeado -en opinión de la Junta Militar- a las universitarios a través de un proceso de concientización impulsado durante el gobierno de la UP. Sin perjuicio de lo anterior la propiaJunta Militar promovió en el mundo académico su propia concepción ideológica de la realidad, aquella del orden, disciplina y progreso, en la cual los académicos enseñan e investigan y los estudiantes estudian y obedecen. 


\section{Nota}

${ }^{1}$ El presente artículo corresponde al proyecto asociativo intitulado "La Universidad de Concepción en un período de cambios históricos: tradición, reforma y transición (1962-1990)”, código 212.064.039-1.0. Financiando por la Vicerrectoría de Investigación y Desarrollo, Universidad de Concepción. 


\section{Bibliografía}

Bresciano, J. (2010), El Tiempo Presente como campo historiográfico. Ensayos teóricos y estudios de casos. Ediciones Cruz el Sur, Montevideo.

Cáceres, L. (2015), De las luchas estudiantiles a las filas de la revolución. Historia del MUI en la Escuela de Servicio Social de la Universidad de Concepción, Escaparate, Concepción.

Flier, P. (2014), Dilemas, apuestas y reflexiones teórico-metodológicas para los abordajes en Historia Reciente, Universidad Nacional de la Plata, Argentina.

Franco, M. y Levín, F. (2007), Historia reciente: perspectivas y desafíos para un campo en construcción. Paidós, Buenos Aires.

Gómez, G. (1995), Origen y desarrollo de la Universidad en Chile, Ediciones de la Universidad de Concepción, Concepción.

Leal, A. (2013), “Así viví el 11 de septiembre hace 40 años”, http:// www.elmostrador.cl/opinion/2013/09/05/asi-vivi-el-11-de-septiembre-hace40-anos/

Monsálvez, D. (2012),’Violencia y represión en un dispositivo local: Concepción, 11 de septiembre de 1973”. Revista de Historia y Geografía, Universidad Católica Silva Henríquez, número 26, junio, p. 57 - 80.

Ídem. (2013),’Chile, la dictadura cívico militar del General Augusto Pinochet y el exilio como dispositivo de control social: el caso de la ciudad de Concepción (1973 - 1976), Revista de Historia y Justicia, número 1, publicación electrónica del Grupo de Investigación Historia y Justicia, Facultad de Filosofía y Humanidades, Universidad de Chile.

Ídem (a 2014), 'Extremistas, enemigos, antipatriotas e indeseables: la legitimidad del Golpe de Estado de 1973 en la prensa escrita de Concepción”. Revista de Historia y Geografía, número 30, Universidad Católica Silva Henríquez, p. 167 - 198.

Ídem(b 2014), Los Bandos Militares en Concepción y Talcahuano: disciplina militar y disciplinamiento social, Concepción, Escaparate.

Ídem(2015), Enemigos, antipatriotas e indeseables: la legitimidad del Golpe de Estado de 1973 en la prensa escrita de Concepción y el origen del Plan Z. Concepción (Chile), Escaparate.

Valderrama, M. (2001), “Renovación socialista y renovación historiográfica”. Programa de Estudios Desarrollo y Sociedad, Documento de Trabajo número 5. 
Valdés, M. (2006), “La Universidad de Concepción el 11 de septiembre de 1973”. Revista de Historia, Universidad de Concepción, año 16, volumen 16 (1), p. 103 a 117.

Ídem, (2012), “El golpe de Estado del 11 de septiembre de 1973 en Concepción (Chile). Las voces de los testigos”, Cuadernos de Historia (U. de Chile), 2012, N 37, p. 159 - 191.

Verdugo, G., Riquelme, L., Soto, V. y Varela, H. (2000), “La Universidad de Pie”. (Documental en video). Trabajo de tesis para optar al título de Periodista, Universidad de Concepción, Departamento de Comunicación Social, Facultad de Ciencias Sociales.

\section{Fuentes:}

Actas de la Honorable Junta de Gobierno. República de Chile. Junta de Gobierno. Secretaria General de Gobierno, septiembre de 1973.

\section{Prensa:}

Diario El Sur, El Diario Color y Diario Crónica de Concepción, septiembre de 1973 a septiembre de 1974.

\section{Entrevistas:}

Fernando AntinaoJelves: Profesor del Instituto de Geografía, Universidad de Concepción, año 1973

Sergio Ramón Fuentealba: Funcionario administrativo Escuela de Periodismo, Universidad de Concepción, año 1973.

Carlos Godoy Rocca: Académico Escuela de Periodismo, Universidad de Concepción, año 1973

Pedro Ilabaca Gajardo: Director del Instituto de Geografía, Universidad de Concepción, año 1973.

Hervi Lagos Cortés: Vicerrector de Finanzas, Universidad de Concepción, año 1973.

Hugo Olea: Periodista y académico Universidad de Concepción, año 1973.

Dagoberto Ulloa: Funcionario Biblioteca Central, Universidad de Concepción, año 1973.

Recibido: 01.06.15

Aceptado: 19.11.15 